\title{
Determinantes de la inversión extranjera directa en América Latina: una visión desde la economía administrativa
}

Gómez Aguirre, Regina; Windler Muñoz, Lia; Massa Roldán, Ricardo

Determinantes de la inversión extranjera directa en América Latina: una visión desde la economía administrativa

Revista Economía y Política, núm. 31, 2020

Universidad de Cuenca, Ecuador

Disponible en: http://www.redalyc.org/articulo.oa?id=571162102005

DOI: https://doi.org/10.25097/rep.n31.2020.03

La Universidad de Cuenca en Ecuador, conserva los derechos patrimoniales (copyright) de las obras publicadas, y favorece y permite la reutilización de las mismas bajo la licencia Creative Commons Atribución-NoComercialCompartirlgual 4.0 Internacional (CC BY-NC-SA 4.0), por lo cual se pueden copiar, usar, difundir, transmitir y exponer públicamente, siempre que: a. Se cite la autoría y fuente original de su publicación (revista, editorial, URL y DOI de la obra). b. No se usen para fines comerciales u onerosos. c. Se mencione la existencia y especificaciones de esta licencia de uso.

Esta obra está bajo una Licencia Creative Commons Atribución-NoComercial-Compartirlgual 4.0 Internacional. 


\section{Determinantes de la inversión extranjera directa en América Latina: una visión desde la economía administrativa}

\section{Factors that determine foreign direct investment in Latin America: an administrative economics vision}

Regina Gómez Aguirre

Tecnológico de Monterrey, México

A01019613@tec.mx

Lia Windler Muñoz

Tecnológico de Monterrey, México

A00369030@tec.mx

\section{Ricardo Massa Roldán}

Programa Interdisciplinario en Regulación y Competencia

Económica, CONACYT-CIDE, México

r.massa@tec.mx

\author{
DOI: https://doi.org/10.25097/rep.n31.2020.03 \\ Redalyc: http://www.redalyc.org/articulo.oa? \\ $\mathrm{id}=571162102005$
}

\section{Resumen:}

El presente trabajo propone una extensión al modelo de la teoría ecléctica de Dunning para explorar el efecto que tienen las variables de la economía administrativa en el comportamiento de los flujos de la inversión extranjera directa (IED) hacia México, Costa Rica, Panamá, Brasil y Perú. Utilizando un modelo de datos panel se estima que para el periodo 2006-2015, variables como el costo de hacer cumplir un contrato, costo de registrar una propiedad, tamaño de la industria bancaria y trabajo en la industria de la agricultura desincentivan la IED hacia países latinoamericanos, al tiempo que elementos relacionados con la apertura comercial la fomentan. En aras de incrementar el flujo de IED hacia países de América Latina, se sugiere diseñar mecanismos que reduzcan los costos de transacción acompañados de políticas públicas que promuevan la competencia dentro del sector bancario local y se persiga la especialización de la mano de obra.

Palabras clave: Inversión, Gobernanza, Latinoamérica, Inversión Extranjera Directa, Datos Panel.

\section{Abstract:}

De present work proposes an extension to Dunning's eclectic theory to explore the effect that variables, with an administrative economics scope, have on the behavior of foreign direct investment (FDI) flows into Mexico, Costa Rica, Panama, Brazil and Peru. Using a panel data model it is estimated that, for the period 2006-2015, variables such as the cost of enforcing a contract, the cost of registering a property, size of the banking industry and labor in the agriculture industry discourage FDI towards Latin American countries, while elements related to trade openness encourage it. In order to increase the flow of FDI into the region, it is suggested to design mechanisms that reduce transaction costs accompanied by public policies that promote competition within the local banking sector and pursue the specialization of labor.

Keywords: Investment, Governance, Latin America, Foreign Direct Investment, Panel Data.

\section{Introducción}

Desde un punto de vista macroeconómico, la inversión extranjera directa (IED) ha sido adoptada como un indicador relevante sobre el grado de crecimiento real y potencial de las economías. Autores como Krugman (1991), Aschauer (1993), Gramlich (1994) y Munnell (1990, 1992) establecen los fundamentos teóricos para que exista una relación positiva entre la inversión, particularmente en proyectos de infraestructura y el desempeño económico de un país. Asimismo, se pueden identificar relaciones positivas entre la entrada 
de capitales extranjeros, la generación de competencia nacional, incrementos de transferencias de capital, presencia de desarrollo tecnológico y el fomento del comercio internacional.

En este contexto, la productividad se considera el aspecto principal que caracteriza el canal de transmisión causal por el cual un aumento en la inversión podría llevar a un incremento en la inversión privada, el empleo y la producción agregada. Si bien actualmente no existe un consenso en la literatura sobre el mecanismo causal que conduce la relación entre IED y el crecimiento económico de los países receptores, la perspectiva generalmente aceptada es que existen efectos positivos, aunque no directos, en dicha relación. En ese sentido, además de explorar si existe un efecto en la productividad, la tendencia general en los estudios actuales consiste en identificar si la inversión muestra efectos indirectos en las variables macroeconómicas que pueden explicarse por los efectos de red y/o efectos de derrame (Banerjee et al., 2012; Vickerman, 2007, 2017).

Los mercados de capitales extranjeros y los inversionistas institucionales son actores cada vez más relevantes en las economías y regiones en desarrollo; tal como lo ilustra Gransow (2015), este es el caso particular de América Latina para países asiáticos, principalmente China. No obstante, esta percepción no parece ser generalizada ya que según la CEPAL (2018), los flujos totales de IED hacia esta región han disminuido durante tres años consecutivos, situación que se explica principalmente por las reducciones en los flujos hacia Brasil, Chile y México. Por otra parte, autores como Della Croce (2011) han manifestado un cambio en la postura de los inversionistas institucionales hacia estrategias de inversión basadas en la asignación de riesgos. El autor sugiere que los gobiernos de países en desarrollo diseñen mecanismos para trabajar más estrechamente con los fondos de pensiones, las compañías de seguros, los mercados de capital y así ampliar el flujo de IED hacia su país. Es ahí donde se presenta un área de oportunidad para América Latina: dada la constante búsqueda de opciones de diversificación de portafolios de inversión, los países de la región deberán generar las condiciones institucionales y comerciales para captar flujos y posteriormente, alcanzar mayores tasas de crecimiento económico.

A partir de esta motivación, resulta fundamental llevar a cabo análisis que identifiquen los factores que influyen en los flujos de IED. Existen modelos, generalmente aceptados e implementados, que buscan explicar el comportamiento de la IED en función de variables macroeconómicas como lo son el modelo Mundell-Fleming, la teoría de tipos de cambio en mercados de capitales imperfectos y el modelo de gravedad. Sin embargo, a la luz de una mayor interconexión financiera entre los países y un endurecimiento de los marcos regulatorios para el comercio y la inversión extranjera, así como una creciente participación de los inversionistas institucionales en el desempeño económico de los países, el estudio de los factores relacionados con elementos microeconómicos (economía administrativa) que derivan en decisiones de flujos de IED, se convierte en un elemento atractivo para analizar. Un modelo que permite, precisamente este análisis, es el desarrollado por Dunning $(1981,1988)$ y que se conoce también como paradigma ecléctico de Dunning, (OLI, por sus siglas en inglés). Este postula una visión general de las motivaciones que influencian la IED a partir de variables propias de la economía administrativa como lo son la gobernanza y la estructura de competencia de los mercados. Aunque el modelo OLI goza de una aceptación teórica generalizada, los estudios empíricos en torno a su implementación son escasos, sobre todo si el objeto de estudio es un país de América Latina.

Es por lo anterior que el presente trabajo tiene dos objetivos principales. El primero es cerrar dicha brecha empírica al presentar un análisis de factores pertenecientes a la rama de la economía administrativa que, dentro del contexto del paradigma OLI, puedan explicar el comportamiento de los flujos de IED hacia cinco países de América Latina: México, Costa Rica, Panamá, Brasil y Perú. El segundo es explorar la existencia de comportamientos heterogéneos de los flujos de IED hacia países de la región, a través de la estimación de un modelo de datos panel. Lo anterior con el fin de abundar en la posibilidad del establecimiento de esfuerzos individuales y/o regionales para el incremento de los flujos de IED hacia los países que la componen. La segunda sección del documento presenta una revisión referente a las motivaciones que se generan, tanto para inversionistas privados como para gobiernos, frente a la decisión de incrementar el flujo de capitales al 
exterior. En la tercera sección se expone un breve resumen de trabajos previos que guardan una relación con el tema, mientras que en la cuarta se describen los aspectos metodológicos y datos utilizados para la estimación a través de modelos de datos panel. Los resultados y la discusión se presentan en la quinta sección. Finalmente, en la sexta sección se establecen conclusiones y se realizan recomendaciones de política pública dentro del contexto del paradigma OLI.

\section{Motivaciones para la inversión extranjera directa}

Los motivos que tienen, tanto los inversionistas privados como los gobiernos, para incrementar el flujo de capital a través de sus fronteras presentan un eje rector que puede estudiarse desde un punto de vista microeconómico. Estas decisiones pueden nacer de condiciones relacionadas con el ciclo del producto (Vernon, 1966), la posibilidad de explotar imperfecciones de mercados extranjeros en comparación a los nacionales (Hymer, 1976), o la generación de oportunidades para el aprovechamiento y explotación de la propiedad, ubicación e internalización de las entidades con participación en el extranjero (Dunning, 1981, 1988).

Particularmente, esta última perspectiva es conocida como el paradigma OLI, o la teoría ecléctica de Dunning y consta de tres determinantes generales de la IED. El primero está constituido por las ventajas de la propiedad ( $O$ por ownership en inglés), el segundo es la ubicación ( $L$ por location en inglés) y el tercero es la internalización ( $I$ por internalization en inglés). La propiedad está relacionada con el grado de pertenencia de los procesos productivos, es decir, quién es "dueño" de las diferentes etapas del proceso productivo. Desde este enfoque, las ventajas de producción y/o transacción resultan determinantes para que los agentes foráneos decidan iniciar o incrementar, el flujo de IED hacia el país/región que las provea. Dichas ventajas se pueden dividir en tres bloques: 1) las que provienen del privilegio exclusivo o acceso a bienes, comúnmente recursos naturales, del país receptor de la IED; 2) las que provienen de la adquisición de activos fijos en el país receptor y las ganancias derivadas de esta y 3 ) las que son consecuencia de la diversificación geográfica.

Por su parte, la ubicación responde a elementos estratégicos, comúnmente relacionados con el aprovechamiento de fallas de mercado observadas en el país receptor y que se refieren al sitio donde se llevará a cabo la producción y/o acceso a mercados para la comercialización de bienes y servicios. En su mayoría se relacionan con la explotación de ventajas en materia de costos (salarios, tarifas y aranceles) y tratamientos fiscales diferenciados. Aunque las ventajas de la ubicación pueden ser fácilmente distinguibles de las de propiedad e internalización, la decisión acerca de la localización presenta una dependencia respecto a la posesión de los bienes y la ruta mediante la cual sus derechos son gestionados, razón por la cual este determinante se estudia generalmente como una consecuencia de los otros dos.

Respecto a las fallas de mercado que influencian la ubicación, se pueden identificar dos grandes grupos: 1) las distorsiones estructurales, es decir, las que surgen de la intervención directa del gobierno en materia de costos y beneficios para producir en diferentes espacios geográficos, y 2) las no estructurales, resultantes de dinámicas propias de las industrias donde pueden obtenerse ganancias con acciones como el arbitraje, apalancamiento, reducción de riesgo de tipo de cambio, manipulación de precios, entre otras. Por último, la internalización está relacionada con la obtención de información y experiencia generada a partir de la presencia en el país receptor y que puede ser utilizada para favorecer el presente y/o futuro desempeño de la entidad de la cual provienen los flujos. Dentro de los elementos más comunes se encuentra la obtención de licencias y permisos, el conocimiento de aspectos empresariales y culturales, así como la construcción y fortalecimiento de relaciones con agentes estratégicos en el país receptor.

Con base en lo anterior, la teoría ecléctica de Dunning puede representarse de la siguiente forma:

$E E D_{i k}=f\left(\right.$ variables propias de $O_{i c}$ variables propias de $L_{i t}$, variables propias de $\left.I_{t 6}\right) \quad$ (1) 
Lo que resulta atractivo de esta perspectiva es que, al comprender el marco general del paradigma OLI, se puede realizar una especificación desde el punto de vista de la economía administrativa.

Es posible vincular directamente los componentes de los tres determinantes con aspectos observables de algún país y así proponerse una relación con el flujo de IED hacia el mismo. En ese sentido, existen dos cuerpos principales de estudios relacionados con la motivación del presente trabajo. El primero estudia el efecto que tienen elementos de gobernanza en la IED hacia países en desarrollo, particularmente los latinoamericanos. Sin embargo, los resultados no son concluyentes respecto a la dirección y significancia de dichos efectos. El segundo se concentra en el estudio empírico de las condiciones con las que el paradigma OLI pueda explicar las motivaciones de la IED hacia América Latina. Pese a considerar elementos de economía administrativa, los trabajos en este segundo grupo son relativamente escasos, por lo que el debate acerca de las variables explicativas relevantes aún está en etapas tempranas. El presente trabajo tiene como objetivo abonar a ese debate.

\section{Paradigma OLI en América Latina}

Dentro del cuerpo de estudios que explora el papel de la gobernanza sobre la entrada de capitales en países latinoamericanos destaca el realizado por Schneider y Frey (1985) quienes generan cuatro modelos para explicar la entrada de capitales en 80 países en vías de desarrollo. Sus conclusiones revelan que la IED hacia dichos países, está influenciado tanto por factores económicos como factores políticos, sin embargo, no abundan en la identificación de los mecanismos causales que le puedan dar explicación. Al respecto, trabajos como los de Pastor y Hilt (1993), Li y Resnick (2003) y Tures (2003) abonan al estudio de componentes políticos ya que señalan que la estructura política del país receptor puede establecerse como principal causa de la atracción de capitales, particularmente cuando se cuenta con un régimen democrático. No obstante, el estudio de Treviño et al. (2002) presenta evidencia de que los riesgos políticos no son un factor relevante si las empresas son incapaces de observar diferencias preponderantes en su desempeño.

Por su parte, Mogrovejo (2005) propone un cambio de perspectiva donde se incorporen condiciones y características intrínsecas de los países, adicionales a los políticos, como determinantes de la IED que reciben. Utilizando variables como el tamaño de mercado, la apertura comercial, el riesgo del país, los costos laborales y las entradas atípicas de inversiones, el autor construye un panel conformado por 19 países con datos de 1990 a 2003. Sus resultados sugieren que los países con mayor producción interna tendrán mayor probabilidad de IED y que variables como el tamaño de mercado, la apertura comercial y el riesgo del país son significativas al explicar el flujo de capitales.

Quazi (2007) incluye la libertad económica de las naciones como variable explicativa y encuentra significancia como determinante de la IED. Los resultados de este estudio son consistentes con la noción generalizada de que un mejor clima empresarial, de inversión, así como la calidad en infraestructura, apertura comercial y retorno en la inversión, impulsarán la entrada de capitales a países latinoamericanos, mientras que la falta de libertad económica tiene un efecto contrario.

Más recientemente, Gomes de Castro, Fernandes y Carvalho (2013) utilizan un modelo de vectores de corrección de error para analizar los principales determinantes de la IED en México y Brasil de 1990 a 2010, encontrando que en Brasil el principal determinante es el tamaño del mercado, mientras que en México es la eficiencia de la liberalización del comercio. Asimismo, Bengoa, Sánchez-Robles y Shachmurove (2012) realizan un estudio de datos panel con 11 países de América Latina para el periodo comprendido entre 1996 y 2012 a través de un modelo de gravedad extendida, revelando que los factores más importantes para la IED son la dotación, la distancia y el costo de la mano de obra de un país objetivo contra el país fuente.

Contrario a lo observado, Montero (2009) encuentra que para los países latinoamericanos el riesgo país tiene poca influencia en la IED, las políticas autocráticas atraen mayor inversión, el tamaño de mercado y la actividad macroeconómica no tienen significancia estadística, y la gobernabilidad es inconsistente como 
predictor de los flujos de IED. Esta conclusión abona a la encontrada por Tumman y Emmert (2004) donde se sugiere que se presenta una mayor atracción de capitales extranjeros cuando existen mayor número de políticas autocráticas en los países receptores. Uno de los resultados más controversiales desde el punto de vista institucional es el presentado por Li y Park (2006) donde se demuestra que los regímenes con menor adhesión a la ley ofrecen mayores incentivos a los inversionistas extranjeros en comparación a los regímenes que son altos en la observancia de leyes. En la misma perspectiva, Jadhav (2012) estudia el rol de factores económicos, políticos e institucionales para atraer IED en los países BRICS del periodo de 2000 a 2009. Se incluyen variables como el tamaño de mercado, apertura al comercio, adquisición de recursos naturales, estabilidad económica, estabilidad política, control de corrupción y estado de derecho. En este caso, sus resultados sugieren que los factores económicos son más significativos que los institucionales y políticos.

Dentro de los estudios más recientes relacionados con elementos institucionales y de gobernanza, Biglaiser y DeRouen (2006) sostienen que las reformas económicas y estructurales no son determinantes de la IED en diferentes países latinoamericanos.

A su vez, Subasat y Bellos (2013) realizan un análisis de datos panel con 21 países de la región en el periodo comprendido entre 1985 y 2008 , en donde se observa que las variables de gobernanza tienen coeficientes negativos, implicando que una mala gobernanza está asociada con altos niveles de IED. En este contexto, altos niveles de corrupción están asociados con motivaciones para incrementar la inversión en América Latina. Finalmente, el trabajo de Staats y Biglaiser (2012) plantea un efecto entre la fuerza judicial y el estado de derecho de países latinoamericanos sobre los flujos que estos reciben de IED, realizando un estudio de datos panel con 17 países en el periodo comprendido entre 1996 y 2007 a través de un modelo de gravedad extendida con efectos fijos. Los resultados obtenidos demuestran que la solidez judicial y el estado de derecho están vinculados positivamente con las entradas de IED en América Latina.

Como puede observarse, si bien la inclusión de variables institucionales, políticas y económicas como determinantes de la IED en América Latina es de gran interés académico y práctico, los resultados no son concluyentes. En gran medida esto se puede explicar en la disociación entre la visión macroeconómica de dichos efectos y su relación con el entendimiento de los procesos de decisión individual de los agentes extranjeros para invertir en la región. En ese sentido, el uso del paradigma OLI para su estudio puede establecerse como el puente entre los componentes microeconómicos de la decisión de inversión y los flujos de capital hacia el país receptor. Al respecto existe un cuerpo limitado de estudios recientes que incorporan la teoría ecléctica de Dunning como marco teórico de referencia. Se destacan trabajos como los de Amal, Tomio y Raboch (2010); y Ramsey, Alvim, Forteza y Micheloni (2010). Sin embargo, el presente trabajo toma su inspiración en el estudio de Padilla y Gomes (2015) donde se utiliza el paradigma OLI y el ciclo de vida del producto de Vernon, como base para determinar los factores de decisión para el flujo de la IED. Encuentran que el componente de internalización de las empresas en países en desarrollo no corresponde a las motivaciones de los modelos de Dunning y Vernon. Para tratar de abundar en el debate de la teoría ecléctica y su relación con la IED en América Latina, el presente trabajo propone una visión de economía administrativa para incorporar variables relacionadas directamente con los determinantes OLI.

\section{Materiales y Métodos}

Para la implementación del presente trabajo se utilizan observaciones anuales del periodo comprendido entre 2006 y 2015 para el caso de cinco países latinoamericanos: México, Costa Rica, Panamá, Brasil y Perú. Con ello se conforma un panel largo de $\overline{N * T=50}$, donde la selección de los sujetos a estudiar sigue un criterio de información disponible para la construcción de un panel balanceado y los datos se obtuvieron de la base de indicadores de desarrollo mundial del Banco Mundial.

El uso de modelos de regresión para datos panel tiene como objetivo principal explorar la presencia de heterogeneidad, es decir, efectos diferenciados entre las unidades de análisis una vez controlado el efecto 
de variables independientes relevantes. En términos meramente matemáticos, la presencia de la misma pendiente para los sujetos de estudio, pero distintas ordenadas al origen.

La raíz de las diferencias entre los individuos puede entenderse desde un punto de vista fijo -características observables de cada uno- o aleatorio -características no observables, pero únicas para el sujeto-. Como lo señala Baltagi (1995), esta construcción permite el estudio de relaciones complejas de comportamiento en comparación a los datos de corte transversal y/o datos de series de tiempo. En ese sentido, la estimación de modelos de datos panel es recomendable para el estudio de fenómenos como el desarrollo tecnológico, impacto de políticas públicas y como se busca ilustrar en el presente trabajo, el comportamiento de flujos comerciales e inversión.

Las técnicas más utilizadas para la estimación de modelos con datos panel son: mínimos cuadrados de coeficientes constantes (MCO Agrupados), mínimos cuadrados considerando efectos heterogéneos con naturaleza fija (efectos fijos) y el que considera efectos heterogéneos con naturaleza aleatoria (efectos aleatorios). El modelo de regresión con MCO agrupados puede expresarse de manera general como:

\section{$\gamma_{i t}=\beta_{0}+\beta_{1} X_{11 t}+\beta_{2} X_{2 l t}+\cdots+\beta_{k} X_{k i t}+u_{t t}$}

Donde el subdíndice 目denota la unidad de ánalisis y el t el periodo de cada observación. La interpretación de los estimadores $\overline{\beta_{1}, \beta_{2} \ldots, \beta_{n}}$ es la convencional, coeficientes de correlación parcial entre las variables independientes y la dependiente. Especial atención se hace en el estimador ßa, ya que al no estar indizado en o en $\mathrm{H}$, se asume como una constante común para todas las unidades de análisis. Es decir, el modelo de MCO agrupado no captura explícitamente efectos de heterogeneidad entre los sujetos y en caso de existir, dicha

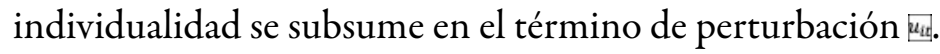

Por otra parte, el modelo de efectos fijos puede expresarse, de manera general, como sigue:

$$
Y_{i t}=\beta_{0 i}+\beta_{1} X_{1 i t}+\beta_{2} X_{2 i t}+\cdots+\beta_{k} X_{k i t}+u_{i t}
$$

A diferencia de MCO agrupados, el término del intercepto $\beta$ incluye ahora algún subíndice para explícitamente estimar efectos heterogéneos que puedan depender estrictamente del sujeto \&, del tiempo $\mathrm{B}$ de ambos 国. Considerando el caso cuando la fuente de la individualidad surge del sujeto, el intercepto puede construirse de manera general como:

$$
\beta_{0 i}=\beta_{0}+\alpha_{1 i} Z_{1}+\alpha_{2 i} Z_{2}+\cdots+\alpha_{n i} Z_{n} \quad \text { (4) }
$$

En este caso, $a_{n}$ puede entenderse como el efecto único de cada individuo debido a la variable o característica observable 2. De tal manera que, en caso de existir efectos heterogéneos, el intercepto de la regresión será distinto para cada sujeto. Finalmente, el modelo de efectos aleatorios mantiene, esencialmente, la misma estructura para la ecuación (3), pero considera que la fuente de heterogeneidad radica en componentes no observables y/o del proceso de muestreo con el cual se construye el panel. En este sentido, el intercepto puede construirse como:

$$
\beta_{0 i}=\beta_{0}+\varepsilon_{i} \quad(5)
$$

Donde 国 representa un término de error aleatorio con media cero y varianza constante, y considera los componentes que puedan influir en una diferencia entre individuos, pero que su efecto no puede controlarse explícitamente en algún estimador. Queda entonces claro que la selección de la herramienta de estimación más apropiada depende tanto de las características de las variables utilizadas, el tamaño de muestra, así como la propiedad de consistencia de los estimadores.

Por lo anterior, resulta relevante caracterizar las variables utilizadas en el presente estudio. Se utilizan seis variables para la construcción del panel, una dependiente (IED) y cinco independientes; todas son transformadas a logaritmo natural para beneficiar la comparabilidad e interpretación de los resultados obtenidos, así como, para reducir la variabilidad del comportamiento de cada una. 
La variable dependiente IED está construida a partir de la información del Fondo Monetario Internacional y reportada por el Banco Mundial, y se define como el flujo neto hacia el país receptor expresado en dólares estadunidenses. El criterio de selección de las 5 variables independientes a utilizar: contratos, propiedad, bancos, agricultura e importaciones, corresponde a su relación con los determinantes del paradigma OLI y un análisis de correlación para evitar potenciales problemas de multicolinealidad entre ellas.

La variable contratos $(\mathrm{CON})$ está definida como el costo de hacer cumplir un contrato como porcentaje de las reclamaciones totales. Esta cifra es obtenida del informe Doing Business reportado por el Banco Mundial. Según la teoría ecléctica, esta variable está vinculada con la internalización $I$ debido a que el costo, hacer cumplir un contrato, refleja el riesgo de invertir en un país, con lo que, la expectativa teórica de esta relación es que exista un signo negativo en el estimador correspondiente. Es decir, a mayor costo de cumplimiento de contrato, menor incentivo para incrementar la IED.

Por su parte, la variable propiedad (PRO) representa el costo de registrar una posesión como porcentaje del valor total de ella. Al igual que la variable CON, es obtenida del informe Doing Business, pero puede ser vinculada al determinante de propiedad, $O$. Se puede esperar que un incremento en los costos de registro de propiedad resulte en un decremento de la IED al implicar más costos asociados a la obtención legal de la pertenencia de los activos correspondientes.

La tercera variable incluida, bancos (BAN), mide el número de sucursales de bancos comerciales por cada 100,000 adultos registrados. Esta información es recabada por el Fondo Monetario Internacional y reportada por el Banco Mundial. Puede verse como un proxy de las ventajas de localización $L$, desde dos ópticas: la de los potenciales consumidores y la de las potenciales ganancias en caso de constituirse como fuentes de financiamiento para los agentes del país receptor. En el primero, una mayor disponibilidad de sucursales bancarias puede vincularse con un incremento en la posibilidad de que los consumidores locales adquieran recursos para la compra de los bienes y/o servicios que la entidad foránea provea. En este caso, podría esperarse un signo positivo entre las variables. Por otra parte, bajo el segundo caso, un mayor número de sucursales de bancos comerciales puede verse como un desincentivo para la IED toda vez que disminuiría la posibilidad de explotar la ventaja de menores precios en un mercado de dinero con una estructura competitiva. Cabe mencionar que, en ambos casos, se hacen fuertes supuestos sobre la penetración del sistema financiero y una industria bancaria con estructura competitiva. Dichos supuestos pueden resultar difíciles de concebir en países de América Latina por lo que el efecto de esta variable puede suponerse ambiguo.

La variable agricultura (AGR) está definida como el porcentaje de personas que trabajan en este sector con relación al total de personal ocupado. Estas actividades incluyen agricultura, caza, pesca y agronomía. La Organización Mundial del Trabajo es la encargada de recabar esta información y compartirla con el Banco Mundial para su publicación. En este caso, su efecto puede entenderse dentro del determinante de localización $L$, en función que pueda verse como una medida del grado de industrialización del país receptor y, con ello, de la oportunidad de explotar oportunidades generadas por la brecha tecnológica entre países. Cómo es planteado por Dunning, se esperaría que los empleos en el sector agricultura presenten una relación inversa con la IED, ya que a las empresas multinacionales estarán motivadas para invertir en procesos productivos de mayor valor agregado donde pudieran generar mayores beneficios potenciales derivados de la brecha tecnológica.

Por último, la variable importaciones (IMP) incluye las transacciones realizadas entre residentes de un país y el resto del mundo por la adquisición de bienes y servicios, expresadas en dólares estadunidenses. El Fondo Monetario Internacional recaba la información de lo publicado en las balanzas de pagos de cada país. Por composición, esta variable representa la apertura comercial, las oportunidades de ventas de productos foráneos, así como el tipo la dinámica de inversión que puede realizarse. En ese sentido, puede ser considerada como una fuente para los tres determinantes del OLI, con un efecto positivo asumido entre los flujos comerciales y el comportamiento de la IED. 
Un hecho estilizado para el trabajo de datos panel y series de tiempo, es la condición de que las variables consideradas dentro del modelo se comporten como procesos de reversión a la media. Esta característica, conocida comúnmente como estacionariedad, determina el tipo de herramienta que resulta apropiada para la estimación de relaciones de corto y/o largo plazo entre las variables utilizadas. Las tres técnicas de estimación descritas anteriormente pueden ser utilizadas ante la presencia de estacionariedad en las variables, es por ello que su verificación resulta fundamental. Como puede apreciarse en la Tabla 1, las seis variables presentan un comportamiento estacionario según la prueba de raíz unitaria de Levin, Lin y Chu (2002). Con ello, los tres tipos de modelos de estimación de datos panel resultan apropiados para el objetivo del presente estudio.

Desde un punto de vista metodológico, es importante rescatar la observación de Sytoian y Filippaios (2008) donde indican que, aunque el paradigma ecléctico es una de las estructuras más influyentes para la investigación empírica de los determinantes de la IED, al ser un modelo de carácter general, se ve limitado para explicar el poder específico de los diferentes tipos de producción en el extranjero o el comportamiento de las empresas. Es por lo anterior que podría considerarse, a priori, que la estimación de MCO agrupados pueda ser más adecuada para capturar el efecto sistemático de las variables seleccionadas sobre el comportamiento de la IED en comparación a los modelos de efectos fijos y efectos aleatorios. Mas aún, el mismo Dunning (2015: 48-49) reconoce que existen limitantes para que el paradigma OLI capture las acciones aleatorias (idiosincráticas) de las empresas, por lo que adicionalmente pudiera descartarse, por diseño, la estimación por el método de efectos aleatorios.

Para poder determinar si las observaciones tanto de Sytoian y Filippaios (2008), como de Dunning (2015), son adecuadas dentro del contexto del presente trabajo, se llevaron a cabo las pruebas de multiplicadores de Lagrange para efectos aleatorios (Breusch-Pagan, Honda, y King-Wu) y la de efectos fijos redundantes (Frestringida). Los resultados de dichas pruebas se presentan en la Tabla 2.

TABLA 1.

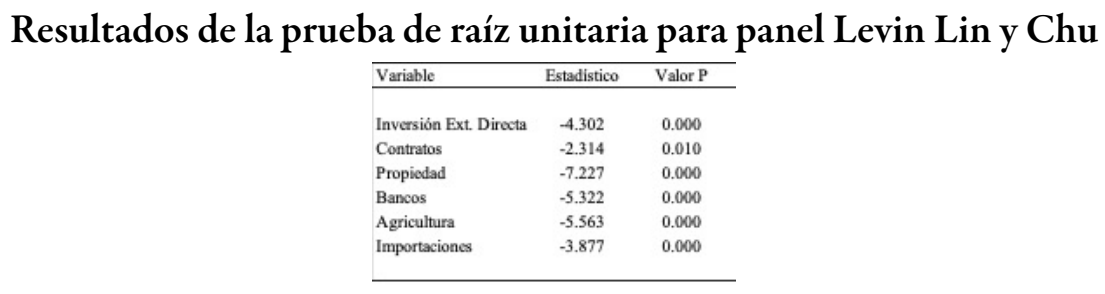

Los autores, utilizando el entorno estadístico R.

TABLA 2.

Resultados de las pruebas para la elección del método de estimación

\begin{tabular}{|c|c|c|c|}
\hline & Individuo & Tiempo & Ambos \\
\hline Breusch-Pagan & 2.743 & 0.375 & 3.117 \\
\hline Valor $\mathrm{P}$ & 0.098 & 0.541 & 0.210 \\
\hline Honda & -1.656 & -0.612 & -1.604 \\
\hline Valor $\mathrm{P}$ & 0.951 & 0.730 & 0.946 \\
\hline King-Wu & -1.656 & -0.612 & -1.717 \\
\hline Valor $\mathrm{P}$ & 0.951 & 0.730 & 0.957 \\
\hline \multicolumn{4}{|c|}{ Prucba F Restrictiva } \\
\hline & Estadistico & GdL & Valor $\mathrm{P}$ \\
\hline F & 0.304 & 4,40 & 0.874 \\
\hline Ji cuad. & 1.497 & 4 & 0.827 \\
\hline
\end{tabular}

Los autores, utilizando el entorno estadístico R.

En lo relacionado con las pruebas de multiplicadores de Lagrange para efectos aleatorios, se puede apreciar que para las tres pruebas y las tres variaciones (individuo, tiempo y ambos) todos los valores $\mathrm{P}$ asociados a los estadísticos se encuentran por encima de un nivel de significancia del 5\%. Es por ello que la hipótesis nula acerca de la no existencia de efectos aleatorios entre las unidades de análisis no se rechaza. Este resultado 
sugiere que el modelo de mínimos cuadrados agrupados es preferido al de efectos aleatorios al no presentarse evidencia de heterogeneidad (con naturaleza aleatoria) entre los países considerados.

En la misma dirección, los valores $\mathrm{P}$ obtenidos de la prueba $\mathrm{F}$ restrictiva indican que el modelo de mínimos cuadrados agrupados es preferido al de efectos fijos, al no rechazar la hipótesis nula de la no existencia de efectos de heterogeneidad con naturaleza determinista. En suma, los efectos heterogéneos de los sujetos de estudio no son significativos. Es decir, los países de América Latina seleccionados no presentan elementos individuales -de carácter determinista o aleatorio- que generen un efecto diferenciado en la determinación de los flujos de IED hacia ellos. Es por esto que se puede justificar la presencia de un comportamiento homogéneo para caracterizar el entorno de los países latinoamericanos de manera regional.

Cabe mencionar que, debido a que en ambas pruebas se prefiere el método de estimación de mínimos cuadrados agrupados, es innecesario llevar a cabo la prueba de Hausman ya que su utilidad está relacionada con encontrar la diferencia entre los estimadores obtenidos a través de los modelos de estimación por efectos aleatorios y efectos fijos como criterio para la selección de alguno de ellos, cuando ambos son preferidos al método de mínimos cuadrados agrupados. Al presentarse el caso contrario, se estima un modelo por MCO agrupados con la siguiente forma funcional:

IED $=\beta_{0}+\beta_{1} C O N_{j}+\beta_{2} P R O_{j}+\beta_{3} B A N_{j}+\beta_{4} A G R_{j}+\beta_{5} I M P_{j}+u_{j} \quad(6)$

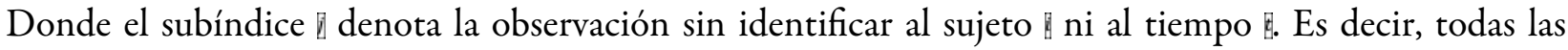
observaciones son consideradas como de corte transversal. Al estimarse por MCO agrupados, se realizan los supuestos convencionales sobre el comportamiento del término de error 匈 y se verifican con las pruebas correspondientes.

\section{ReSULTADOS Y DISCUSIÓN}

Siguiendo la metodología expuesta en Wooldridge (2010) para la estimación de MCO agrupados, se obtienen los resultados presentados en la Tabla 3. Por su parte, la Tabla 4 muestra los resultados de las principales pruebas para verificar los supuestos de autocorrelación y heteroscedasticidad. Para el caso de las pruebas de autocorrelación serial y contemporánea, la hipótesis nula de no autocorrelación no puede rechazarse ya que se observa que los valores $\mathrm{P}$ obtenidos superan un nivel de significancia del $5 \%$ para las tres pruebas realizadas. Del mismo modo, la hipótesis nula de varianza constante -homoscedasticidad-tampoco es rechazada ya que para las dos versiones de la prueba Breusch-Pagan presentadas, los valores $\mathrm{P}$ obtenidos son 0.066 y 0.184 respectivamente.

Después del cumplimiento de las pruebas de verificación de supuestos correspondientes se concluye que los estimadores son adecuados para describir el efecto que tienen las variables independientes en la IED de la región.

TABLA 3.

\section{Resultados de la estimación por MCO agrupados}

\begin{tabular}{|c|c|c|c|}
\hline Variables & Coeficiente & Error Est. & Estadistico t \\
\hline Intercepto & 6.420 & 1.136 & 5.65 \\
\hline Contratos & -0.621 & 0.162 & -3.83 \\
\hline Propiedad & -1.002 & 0.164 & -6.11 \\
\hline Bancos & -0.928 & 0.164 & -5.67 \\
\hline Agricultura & -0.874 & 0.259 & -3.38 \\
\hline Importaciones & 1.003 & 0.040 & 25.37 \\
\hline R cuad. & 0.965 & & \\
\hline R cuad. Aj. & 0.961 & & \\
\hline Estadistico $F$ & 245.69 & & \\
\hline Estadistioo D-W & 1.906 & & \\
\hline
\end{tabular}


Los autores, utilizando el entorno estadístico R.

TABLA 4.

Cumplimiento de los supuestos del modelo de MCO agrupados

\begin{tabular}{lcc}
\hline \multicolumn{3}{l}{ Pruebas de autocorrelación serial y contemporánea } \\
\hline & Estadistico & Valor P \\
\hline Breusch-Godfrey/Wooldridge & 16.021 & 0.099 \\
& & \\
Breusch-Pagan & 16.828 & 0.078 \\
Pesaran & 1.527 & 0.127 \\
& & \\
\hline & & \\
\hline & & \\
\hline & Estadistico & Valor P \\
\hline & 10.354 & 0.066 \\
Breusch-Pagan & 7.538 & 0.184 \\
Breusch-Pagan estudentizado & & \\
\hline
\end{tabular}

Los autores, utilizando el entorno estadístico R.

Es importante recordar que, al haber transformado las series a logaritmos naturales, la interpretación de los coeficientes es a través del concepto de elasticidades o sensibilidades. Asimismo, las expectativas del signo en el coeficiente de la variable contratos se cumplen al obtenerse un resultado significativo y negativo. En este sentido, como se propone en la teoría ecléctica un incremento porcentual en el costo de hacer cumplir los contratos, genera en promedio, una reducción de $0.621 \%$ en la IED en la región. Del mismo modo, el signo del coeficiente de la variable propiedad es negativo indicando que el incremento porcentual en el costo para registrar una propiedad está asociado, en promedio, a una reducción del $1 \%$ en la IED aproximadamente.

El signo obtenido para el coeficiente de la variable bancos sugiere una posible visión de competencia respecto a la proveeduría de capital en el contexto local. Es decir que mientras más sucursales bancarias existen, menor es la oportunidad de los agentes foráneos para explotar la estructura de costos financieros para su beneficio. Puntualmente, un incremento porcentual en el número de sucursales bancarias está ligado a una reducción, en promedio, de la IED en casi $0.9 \%$ para la región.

Del mismo modo que las tres variables anteriores, el coeficiente para agricultura presenta un signo negativo, resultado en línea con la teoría ecléctica. Particularmente, este efecto puede estar explicado por la búsqueda de mayores grados de especialización como elemento para decidir el país en donde invertir. Es decir, en cuanto a las ventajas de localización, una nación con infraestructura centrada en los sectores secundarios y terciarios, pero que no tenga mano de obra calificada, puede resultar en una reducción en la consideración para invertir derivado de la necesidad de una sobreinversión en programas de capacitación, entre otros componentes estrictamente no directos. Para el caso de la presente estimación, un incremento porcentual en la población que labora en actividades relacionadas a la agricultura está asociado a una reducción promedio de casi $0.87 \%$ en la IED.

Finalmente, para el caso del coeficiente de la variable de importaciones el signo presentado es positivo. Este efecto puede explicarse por la posibilidad y reconocimiento de apertura comercial que puede ser considerada tanto una ventaja de localización como de internalización. Por lo tanto, un incremento porcentual en el flujo de importaciones está ligada a un incremento de magnitud similar en la IED.

\section{CONCLUSIONES Y RECOMENDACIONES}

En conjunto, de las variables incluidas en el presente análisis, los costos asociados al registro de la propiedad resultan ser el principal determinante para la salida de IED de los países estudiados, mientras que el incremento en el flujo de las importaciones se reconoce como el único factor que presenta una relación positiva con la entrada de IED. En este sentido, cuando se considera la relación conceptual que tiene la libertad económica y apertura comercial en el flujo de IED hacia la región, resulta interesante observar que en 
términos de signos, nuestros resultados son similares a los presentados en el trabajo de Quazi (2007). El autor concluye que existe un efecto positivo entre la apertura comercial y la entrada de capitales a la región. Esta misma dirección es la que obtenemos para el caso de la variable importaciones. Dicha comparación creemos que es válida ya que ambas variables esencialmente caracterizan la posibilidad de llevar a cabo transacciones de venta en los países receptores, explotando entonces las ventajas de propiedad, localización e internalización dentro del contexto del paradigma OLI. Por otra parte, el autor señala que existe un coeficiente negativo entre la falta de libertad económica y la entrada de capitales a los países receptores. Bajo esa línea, el signo obtenido en el presente trabajo es consistente para las variables tamaño de la industria bancaria, costos de hacer cumplir los contratos y los de registro de propiedades. Aunque, por definición, se tratan de variables distintas, se puede argumentar que la relación subyacente está vinculada al ambiente empresarial y las motivaciones relacionadas con potenciales barreras administrativas y/o de competencia para el desempeño de actividades comerciales dentro del país receptor.

El presente estudio extiende la literatura, puntualmente en referencia con los trabajos de Amal, Tomio y Raboch (2010); Subasat y Bellos (2013); y, Padilla y Gomes (2015), sobre la relación entre la gobernanza de un país y la estructura institucional, laboral y comercial con la que cuenta, bajo una visión de economía administrativa. Nuestros resultados son consistentes con la noción presente en los tres trabajos respecto a que un mejor clima empresarial --caracterizado por bajos costos de registro de propiedad y de cumplimiento de contratos--, así como la apertura comercial y la competitividad de las industrias impulsan la entrada de capitales a países latinoamericanos. Es por ello que, desde nuestros resultados, es posible detectar implicaciones directas sobre el diseño de políticas públicas y políticas económicas pertinentes que busquen fomentar el flujo de la IED hacia el país, particularmente en las siguientes áreas:

Costos de transacción: La relación inversa presentada entre los costos de transacción en estos países -costos de hacer cumplir un contrato y costos de registro propiedad-- y el flujo de IED resulta preocupante en dos sentidos: el primero, ya documentado con anterioridad, es el impacto directo sobre la salida de IED ante la reducción de explotar las posibilidades de localización y el segundo, potencialmente con mayores repercusiones sociales y económicas, es la posibilidad que en países con altos grados de corrupción, el incremento en costos genere incentivos perversos hacia las actividades ilícitas. Por lo tanto, una sugerencia natural, es la de buscar mecanismos para la reducción de costos, centrados en hacer eficientes tanto los procesos gubernamentales como los notariales.

Contraposición de industrias: Si bien, para el caso del sector bancario los resultados presentados pueden interpretarse como que un sector fuerte y competitivo genera desincentivos para la IED, los supuestos sobre los que descansa -alto grado de inclusión financiera y desarrollo de productos- son raramente encontrados en países de la región. Por lo tanto, la presencia de un mayor número de sucursales no implicará necesariamente un sector bancario competitivo.

Por lo antes mencionado, se propone diseñar e implementar estrategias que promuevan la competencia dentro del sector bancario local. Asimismo, a la luz de la relación inversa entre el trabajo en el sector de la agricultura y la IED, se sugiere realizar inversión local para el fomento de la agricultura de manera paralela a la implementación de mejoras en materia de educación, de tal forma que se pueda perseguir la especialización de la mano de obra sin dañar el desempeño del sector.

\section{REFERENCIAS BIBLIOGRÁFICAS}

Amal, M., Tomio, BT., y Raboch, H. (2010). Determinants of Foreign Investment in Latin America. Journal of Globalization, Competitiveness \& Governability, 4(3), 116-133.

Aschauer, D.A. (1993). Genuine Economic Returns to Infrastructure Investment. Policy Studies Journal, 21(2), 380-90.

Baltagi, B. (1995). Econometric Analysis of Panel Data. John Wiley and Sons. New York. 
Regina Gómez Aguirre, et al. Determinantes de la inversión eXtranjera directa en América Latina: u...

Banerjee, A., Duflo, E., \& Qian, N. (2012). On the Road: Access to Transportation Infrastructure and Economic Growth in China. NBER Working Paper No. 17897.

Bengoa, M., Sánchez-Robles, B., y Shachmurove, Y. (2012). Latin America's FDI patterns: A panel data gravity model to assess the role of regional integration agreements. Conference Paper.

Biglaiser, G. y DeRouen, K. (2005). Economic Reforms and Inflows of Foreign Direct Investment in Latin America. Latin American Research Review, 41(1), 51-75.

Comisión Económica para América Latina y el Caribe (CEPAL). (2018). Foreign Direct Investment in Latin America and the Caribbean 2018, LC/PUB.2018/13-P, Santiago.

Della Croce, R. (2011). Pension Funds Investment in Infrastructure: Policy Actions. OECD Working Papers on Finance, Insurance and Private Pensions, No. 13, OECD Publishing, Paris.

Dunning, J. (1981). Explaining the International Direct Investment Position of Countries: Towards a Dynamic or Developmental Approach. Weltwirtschaftliches Archiv, 117(1), 30-64.

Dunning, J. (1988). The Eclectic Paradigm of International Production: A Restatement and Some Possible Extensions. Journal of International Business Studies, 19(1), 1-31.

Dunning, J. (2015). Explaining International Production. Routledge Revivals. New York.

Jadhav, P. (2012). Determinants of foreign direct investment in BRICS economies: Analysis of economic, institutional and political factor. Procedia - Social and Behavioral Sciences, 37, 5-14.

Gomes de Castro, P., Fernandes, E. y Carvalho, A. (2013). The Determinants of Foreign Direct Investment in Brazil and Mexico: An Empirical Analysis. Procedia Economics and Finance, 5, 231-240,

Gramlich, E.M. (1994). Infrastructure Investment: A Review Essay. Journal of Economic Literature, 32(3), 1176-96.

Gransow, B. (2015). Chinese Infrastructure Investment in Latin America--An Assessment of Strategies, Actors and Risks. Journal of Chinese Political Science, 20(3), 267-287.

Hymer, S. H. (1976). The International Operations of National Firms: A Study of Direct Foreign Investment. MIT Press. Cambridge.

Krugman, P. (1991). Increasing Returns and Economic Geography. Journal of Political Economy, 99(3), 483-99.

Levin, A., Lin, C.F., y Chu, C.S.J. (2002). Unit root tests in panel data: asymptotic and finite-sample properties. Journal of Econometrics, 108(1), 1-24.

Li, Q. y Resnick, A. (2003). Reversal of Fortunes: Democratic Institutions and Foreign Direct Investment Inflows to Developing Countries. International Organization, 57(1), 175-211.

Li, S. y Park, S. H. (2006). Determinants of Locations of Foreign Direct Investment in China. Management and Organization Review, 2, 95-119.

Mogrovejo, J. (2005). Factores determinantes de la inversión extranjera directa en algunos países de Latinoamérica. Revista Latinoamericana de Desarrollo Económico, 5, 51-82.

Montero, A. (2009). Political Governance and Macroeconomic Variables in Determining Foreign Direct Investment. Latin American Research Review, 44(1) 195-198.

Munnell, A. H. (1990). How Does Public Infrastructure Affect Regional Economic Performance? New England Economic Review, Sep-Oct, 11-33.

Munnell, A. H. (1992). Policy Watch: Infrastructure Investment and Economic Growth. Journal of Economic Perspectives, 6(4), 189-198.

Padilla, R. \& Gomes, C. (2015). Determinantes de la salida de IED y efectos en el país emisor: Evidencia de América Latina. CEPAL - Serie de estudios y perspectivas (166).

Pastor, M. y Hilt, E. (1993) Private investment and democracy in Latin America. World Development, 21(4), 489-507.

Quazi, R. (2007). Foreign Direct Investment in Latin America: A Panel Regression Study. The International Journal of Business and Finance Research, 1(1), 59-67.

Ramsey, J.R., Alvim, F.M., Forteza, J.H., y Micheloni, J. (2010). International Value Creation: An Alternative Model for Latin American Multinationals. Globalization, Competitiviness \& Governability, 4(3) 62-83. 
Schneider, F. y Frey, B.S. (1985). Economic and political determinants of foreign direct investment. World Development, 13(2), 161-175.

Staats, J.y Biglaiser, G. (2012). Foreign Direct Investment in Latin America: The Importance of Judicial Strength and Rule of Law. International Studies Quarterly, 56(1), 193-202.

Subasat, T. y Bellos, S. (2013). Governance and Foreign Direct Investment in Latin America: A panel gravity model approach. Latin American Journal of Economics, 50(1), 107-131.

Sytoian, C. y Filippaios, F. (2008). Dunning's eclectic paradigm: A holoistic, yet context specific framework for analysing the determinants of outward FDI: Evidence from international Greek Investmemts. Institutional Bussines Review, 17(3), 349-367.

Treviño, L., Daniels, J., Arbelaez, H. y Upadhyaya, K. (2002). Market reform and foreign direct investment in Latin America: Evidence from an Error Correction Model. The International Trade Journal, 16(4), 367-39.

Tumman, J. P. y C. F. Emmert (2004). The political economy of U.S. foreign direct investment in Latin America: a reappraisal. Latin American Research Review, 39(3), 9-29.

Tures, J. (2003) The impact of instability and institutions on US foreign direct investment in developing areas. Conflict, Security \& Development, 3(2), 163-183.

Vernon, R. (1966). International Investment and International Trade in the Product Cycle. The Quarterly Journal of Economics, 80(2), 190-207.

Vickerman, R. (2007). Cost-Benefit Analysis and Large-Scale Infrastructure Projects: State of the Art and Challenges. Environment and Planning B: Planning and Design, 34(4), 598-610.

Vickerman, R. (2017). Wider Impacts of Megaprojects: Curse or Cure? in B. Flyvbjerg (ed.), The Oxford Handbook of Megaproject Management, Oxford University Press, Oxford.

Wooldridge, J.M. (2010). Econometric Analysis of Cross Section and Panel Data. MIT Press. Cambridge.

La Universidad de Cuenca en Ecuador, conserva los derechos patrimoniales (copyright) de las obras publicadas, y favorece y permite la reutilización de las mismas bajo la licencia Creative Commons AtribuciónNoComercial-CompartirIgual 4.0 Internacional (CC BY-NC-SA 4.0), por lo cual se pueden copiar, usar, difundir, transmitir y exponer públicamente, siempre que: a. Se cite la autoría y fuente original de su publicación (revista, editorial, URL y DOI de la obra). b. No se usen para fines comerciales u onerosos. c. Se mencione la existencia y especificaciones de esta licencia de uso.

CC BY-NC-SA

\section{INFORMACIÓN ADICIONAL}

Código JEL:: C23, F21, O16, M21 